\title{
Experiencia con el uso de plasma rico en plaquetas autólogo en el tratamiento de las úlceras del pie diabético
}

Use of platelet rich plasma in the treatment of diabetic foot ulcers. Report of the experience

Experiência com o uso de plasma autólogo rico em plaquetas no tratamento de úlceras do pé diabético

Pablo Orellanoํㅜ, Matías Torres Negreira², Agustín Colombo³, Natalia Lamela4, Daniela Saliwonczyk ${ }^{5}$, Maximiliano Berro ${ }^{6}$, Gabriela Sierra², Ismael Rodríguez ${ }^{7}$, Lilian Vucovich ${ }^{8}$

\section{Resumen}

Las úlceras de pie diabético (UPD) generan un alto costo para el paciente y el sistema de salud. Una deficiente vascularización, la inhibición de la generación de factores de crecimiento y la migración celular, entre otros factores, influyen en su cronicidad. Los factores de crecimiento derivados de las plaquetas (PDGF) estimulan la quimiotaxis, la neovascularización y la regeneración tisular.

Objetivo: evaluar la efectividad de una formulación de plasma rico en plaquetas autólogo (PRP) como una modalidad complementaria para el tratamiento de UPD, valorando su seguridad y efectos adversos.

Materiales: estudio observacional. Se aplicó PRP a seis pacientes con UPD crónicas, Wagner II, III, con una media en el retraso de la cicatrización de 94 semanas. Se administró mediante punción y como gel sobre la lesión una vez por semana hasta el cierre de la úlcera o durante12 semanas. Las UPD se analizaron con respecto al área y su progresión mediante Mobile Wound Analyzer (MOWA), software de análisis de imágenes de úlceras. El porcentaje de reducción del área se calculó entre la medición inicial y la semana 12. Como criterio de mejoría se eligió una reducción $\geq 70 \%$ de la lesión.

Resultados: predominó el sexo masculino con una media de edad de 53 años $\pm 7,3$. Luego de 12 semanas de tratamiento, se reportó una reducción media del tamaño de la úlcera de 78\%, con dos cicatrizaciones completas y dos en $99 \%$. Un pa-

1. Ex Profesor Adjunto de Endocrinología y Metabolismo, Unidad de Pie Diabético, Hospital de Clínicas, Facultad de Medicina. Universidad de la República. Uruguay.

2. Posgrado de Endocrinología y Metabolismo, Unidad de Pie Diabético, Hospital de Clínicas, Facultad de Medicina. Universidad de la República. Uruguay.

3. Asistente de la Cátedra de Hemoterapia y Medicina Transfusional del Hospital de Clínicas, Facultad de Medicina. Universidad de la República. Uruguay

4. Ex Asistente de la Cátedra de Hemoterapia y Medicina Transfusional del Hospital de Clínicas, Facultad de Medicina. Universidad de la República. Uruguay

5. Residente de la Cátedra de Hemoterapia y Medicina Transfusional del Hospital de Clínicas, Facultad de Medicina. Universidad de la República. Uruguay

6. Profesor Adjunto de la Cátedra de Hemoterapia y Medicina Transfusional del Hospital de Clínicas, Facultad de Medicina. Universidad de la República. Uruguay.

7. Prof. Director de la Cátedra de Hemoterapia y Medicina Transfusional del Hospital de Clínicas, Facultad de Medicina. Universidad de la República. Uruguay.

8. Prof. Agrdo. Directora de la Carrera de Podología EUTM, Unidad de Pie Diabético, Facultad de Medicina, Universidad de la República, Uruguay Unidad de Pie Diabético. Servicio de Hemoterapia y Medicina Transfusional, Escuela de Tecnología Médica. Carrera de Podología. Hospital de Clínicas. Montevideo. Uruguay.

Correspondencia: Dr. Pablo Orellano. Correo electrónico: porellanoc@gmail.com

Los autores declaran no presentar conflictos de intereses.

Aprobado por el Comité de Ética del Hospital de Clínicas

Recibido: 18/11/2020

Aprobado: 8/2/2021

Attribution-NonCommercial 4.0 International (CC BY-NC 4.0) 
ciente recibió una amputación luego de presentar infección no controlada.

Conclusión: la aplicación del PRP mejoró la regeneración de tejidos, acortando la duración de la úlcera, promoviendo su curación sin efectos adversos, al tiempo que eliminó la necesidad de procedimientos de manejo en una úlcera crónica.

Palabras clave: Pie diabético

Úlcera del pie

Factor de crecimiento derivado de plaquetas

Plasma rico en plaquetas

Key words: Diabetic foot

Foot ulcer

Platelet-derived growth factor

Platelet-rich plasma

\section{Introducción}

Más de 150 millones de personas en todo el mundo padecen diabetes, $\mathrm{y}$ todos corren el riesgo de desarrollar una úlcera de pie diabético (UPD) de diverso grado, más del $15 \%$ realmente desarrollará dichas úlceras ${ }^{(1,2)}$. La mala vascularización y la matriz tisular inadecuada a menudo afectan el manejo de las úlceras diabéticas, entre varios factores. Diversos tratamientos son utilizados para manejar las UPD, los métodos empleados tradicionalmente incluyen cambios rigurosos de apósito con observación cuidadosa, desbridamiento del tejido necrótico, control de infecciones y reducción de la presión sobre el pie diabético ${ }^{(2-4)}$.

La revascularización de las extremidades inferiores también es considerada en algunos casos ${ }^{(4)}$. La falla en el resultado de los tratamientos puede provocar amputaciones hasta en un $20 \%$ de las UPD infectadas, con aumento de la morbimortalidad y alteración en la calidad de vida del paciente ${ }^{(5)}$.

Determinar los métodos de tratamiento para evitar las amputaciones en todos los pacientes es un problema de calidad de vida y un desafío financiero para los sistemas de salud. Las terapias celulares emergentes, como el plasma rico en plaquetas (PRP), pueden tener un papel complementario en el tratamiento de las UPD.

Han sido múltiples las experiencias clínicas en relación con el empleo de PRP autólogo en el ámbito de la otorrinolaringología, cirugía plástica, medicina deportiva, traumatología ${ }^{(6)}$. Ensayos clínicos han investigado la eficacia del PRP en la cicatrización de heridas crónicas $^{(7-12)}$. Una revisión de Cochrane observó que el PRP puede mejorar la curación en UPD en dos estudios clínicos aleatorizados ${ }^{(13)}$. Otra revisión actual también informa del beneficio ${ }^{(14)}$. Las proteínas contenidas en los gránulos alfa poseen una fuerte influencia sobre los fenómenos reparadores de las heridas. Entre dichas proteínas se encuentran los factores de crecimiento derivados de plaquetas (PDGF), el factor de crecimiento transformante beta (TGF- $\beta$ ), el factor plaquetario 4 (FP4), interleuquina 1 (IL-1), el factor angiogénico derivado de plaquetas (PDAF), el factor de crecimiento endotelial vascular (VEGF), el factor de crecimiento epidérmico (EGF), el factor de crecimiento endotelial derivado de plaquetas (PDEGF), el factor de crecimiento celular epitelial (ECGF), el factor de crecimiento tipo insulina (IGF), osteocalcina, osteonectina, fibrinógeno, fibronectina y trombospondina (TSP). Algunas de estas proteínas se encuentran ausentes en heridas crónicas que no sanan adecuadamente ${ }^{(15)}$.

En la UPD crónica se alteran las etapas de cicatrización, presentando una fase inflamatoria prolongada, defectos en la remodelación de la matriz extracelular, formación de radicales libres e inhibición de la generación de factores de crecimiento celular y de la migración celular. El PRP puede definirse como un volumen de plasma autólogo que contiene una concentración de plaquetas superior al nivel basal.

Luego de la aplicación de PRP el 95\% de los factores de crecimiento se liberan en el lapso de una hora. Adicionalmente, la síntesis y secreción de proteínas dura entre 5 a 10 días más. Luego, los macrófagos que llegan al foco debido al crecimiento vascular promovido por las plaquetas, asumen la regulación de la reparación tisular mediante la secreción de sus propios factores. El uso de PRP autólogo es un tratamiento efectivo y seguro, con escasos efectos adversos leves ${ }^{(16,17)}$. Aún no se ha determinado cuál es el mejor método de preparación y conservación de PRP para aplicaciones clínicas $^{(18)}$. 


\section{Objetivo general}

Evaluar la capacidad del PRP autólogo para cicatrizar UPD crónicas refractarias como una modalidad complementaria de tratamiento, valorando su seguridad y efectos adversos. Evaluar el porcentaje de reducción del área de la herida en un plazo de 12 semanas.

\section{Material y método}

Estudio abierto, prospectivo, observacional. Pacientes con UDP provenientes del sistema de salud público, derivados del primer nivel de atención, policlínica de cirugía, emergencia e internación y que se asisten en la Unidad de Pie Diabético del Hospital de Clínicas, junto al Servicio de Hemoterapia y la Escuela de Tecnología Médica, área de Podología, entre marzo de 2018 y diciembre de 2019. Luego de calificar para los criterios de inclusión (tabla 1), se enrolaron UDP crónicas estadificadas con la clasificación de Wagner como grado II, III, con al menos 12 semanas de retraso en su cicatrización ${ }^{(19,20)}$. Se registraron datos demográficos, como edad y sexo (tabla 2), historial de lesiones previas, antecedentes comórbidos y uso de fármacos que interfirieran con el proceso de reparación (corticoides, anticoagulantes, quimioterapia). Los pacientes seleccionados tuvieron un período de preinclusión de 12 semanas con tratamiento estándar, en base a desbridado y suero fisiológico (SF) con un resultado pobre en cuanto a disminución del diámetro de la úlcera. Se evaluó la infección clínica y la terapia antibiótica empírica apropiada se inició al diagnóstico, controlando que ésta hubiera remitido antes del tratamiento con PRP. Se valoró la perfusión con estudio Doppler en conjunto con cirugía vascular. Para confirmar un recuento plaquetario normal solicitamos hemograma, función renal y hepática por métodos convencionales, así como serología de enfermedades transmisibles HIV 1-2, HTLV 1-2, VDRL, Chagas, hepatitis B Core, Ag de superficie y hepatitis C. Los pacientes continuaron con el control y las prescripciones para su diabetes dirigidas por endocrinólogo tratante. Hemoglobina A1c (HbA1c), se solicitó en el seguimiento (medida por inhibición de aglutinación de látex equipamiento DCA 2000 Bayer-Siemens). En cada consulta se realizó el control clínico y estado general del paciente, glucemia capilar, valoración de la úlcera y el pie, pesquisando la presencia de efectos adversos de la aplicación de PRP: alergias, dermatitis, inflamación, infección, dolor en el sitio de aplicación o cicatriz fibro$\mathrm{sa}^{(16)}$. Cumplieron con los criterios de inclusión y exclusión para la elegibilidad del estudio seis pacientes con antecedentes de amputación menor y diverso grado de enfermedad vascular asintomática ${ }^{(21)}$. Se excluyeron seis pacientes con infección no controlada.
Tabla 1. Criterios para la selección de pacientes.

Criterios de inclusión
- Diabéticos que se atienden en UPD del Hospital de Clínicas.
- Mayores de 18 años.
- Que aprueben el consentimiento informado.
- nortadores de úlceras: neuropáticas, isquémicas y/o
- Tiempo de evolución de la úlcera: mayor a 12 semanas.
- No cierre de la úlcera luego de 12 semanas de tratamiento
estándar.
- Pacientes con patología hepática, inmune, o coagulopatías.
- Pacientes portadores de enfermedades malignas o terminales.
- Pacientes que reciben tratamientos inmunosupresores o
radioterapia. Pacientes con abuso de alcohol o drogas.
- Pacias antes de la aplicación restableciéndose su uso posterior al
- Procedimiento.
- Pacientes embarazadas.
- Paciente conten IMC < 18.

\section{Obtención y aplicación de PRP}

La fase de centrifugación se realizó por médico hemoterapeuta entrenado en la obtención de hemocomponentes, para posibilitar el máximo rendimiento del producto. Se les extrajo un volumen de sangre total de 15 a 50 $\mathrm{ml}$, las muestras se obtuvieron mediante sistema de tubos vacutainer en tubo citratado a 3,8\%. Mediante un proceso que utiliza el principio de separación celular por centrifugación diferencial, se obtuvo PRP en estrictas condiciones de asepsia en cámara de flujo laminar para evitar la contaminación del producto en el laboratorio de hemoterapia (figura 1). Se realizó el control microbiológico de cada producto de PRP, sin obtener desarrollo microbiológico. Se realizó una primera centrifugación de la sangre total a $22{ }^{\circ} \mathrm{C}$, a una velocidad de $1.800 \mathrm{rpm}$ en 5 minutos, separando los glóbulos rojos 
Tabla 2. Características de los pacientes.

\begin{tabular}{lccccc}
\hline Pacientes $^{1}$ & Sexo & Edad (años) & HbAlc (\%) & $\begin{array}{c}\text { Clasificación } \\
\text { de Wagner }{ }^{2}\end{array}$ & $\begin{array}{c}\text { Tiempo de evolución de } \\
\text { la úlcera (semanas) }\end{array}$ \\
\hline 1 & Masculino & 60 & 9 & 3 & 240 \\
2 & Femenino & 48 & 11 & 3 & 12 \\
3 & Masculino & 49 & 7 & 2 & 144 \\
4 & Masculino & 56 & 8 & 3 & 48 \\
5 & Masculino & 42 & 8 & 3 & 96 \\
\hline
\end{tabular}

1. Todos presentaban una amputación menor previa y enfermedad vascular periférica.

2. Wagner FW. The dysvascular foot: a system for diagnosis and treatment. Foot Ankle 1981:264-122.

HbA1c: hemoglobina glicosilada.

Tabla 3. Porcentaje de reducción del área de la úlcera luego de PRP.

\begin{tabular}{|c|c|c|c|c|}
\hline \multirow[t]{2}{*}{ Pacientes } & \multicolumn{2}{|c|}{ Área $\mathrm{cm}^{2}$} & \multirow[t]{2}{*}{ \% reducción } & \multirow[t]{2}{*}{ Forma de aplicación } \\
\hline & Inicial & Final & & \\
\hline 1 & 6,0 & 1,5 & 75 & PRP-PG \\
\hline 2 & 9,7 & 9,7 & 0 & PRP-PG \\
\hline 3 & 2,9 & 0 & 100 & PRP-PG \\
\hline 4 & 11,7 & 0,1 & 99 & PRP-G \\
\hline 5 & 4,2 & 0 & 100 & PRP-G \\
\hline 6 & 21,7 & 0,2 & 99 & PRP-G \\
\hline$x$ & $99,36( \pm 6,29)$ & $1,91( \pm 3,52)$ & 78,83 & \\
\hline
\end{tabular}

PRP: plasma rico en plaquetas. PRP-PG: punción-gel. PRP-G: gel. x: media.

del plasma con las plaquetas; éste se sometió a una segunda centrifugación a $22^{\circ} \mathrm{C}$, a una velocidad de 2.500 rpm, durante 8 minutos, descartando los dos tercios superiores del plasma pobre en plaquetas, obteniendo así el PRP. El producto fue destinado a inyectarse directamente o a la aplicación como gel plaquetario. En el PRP, nuestro objetivo fue llegar a concentrar las plaquetas al menos tres veces su valor basal ${ }^{(22,23)}$. La cantidad de PRP utilizado se definió según el tamaño y la profundidad de la úlcera. Decidimos no activar previamente el producto inyectable, sabiendo que la activación se realiza al contacto de las plaquetas con el tejido cruento de los bordes y el fondo desbridado de la úlcera. El gel se produjo activando el PRP con gluconato de calcio a $10 \%$ y dejándolo a $37^{\circ} \mathrm{C}$ hasta obtener su gelificación. Se aplicó PRP mediante inyección y como gel sobre la úlcera (figura 2) en tres pacientes consecutivos y únicamente como gel en los restantes tres pacientes (tabla 3). Luego de la aplicación, se realizó una compactación suave con apósitos de gasa seca para cubrir la superficie y absorber cualquier descarga. El apósito se retiró a las 24 horas continuando con curaciones diarias en domicilio con SF. La aplicación de PRP se realizó una vez por semana hasta el cierre de la úlcera o hasta completar 12 semanas. Antes de aplicar el PRP, la UPD se preparó utilizando un desbridamiento cuidadoso y riego vigoroso con SF. Se realizó el registro fotográfico y medida del área de la UPD al inicio del estudio y pre- 


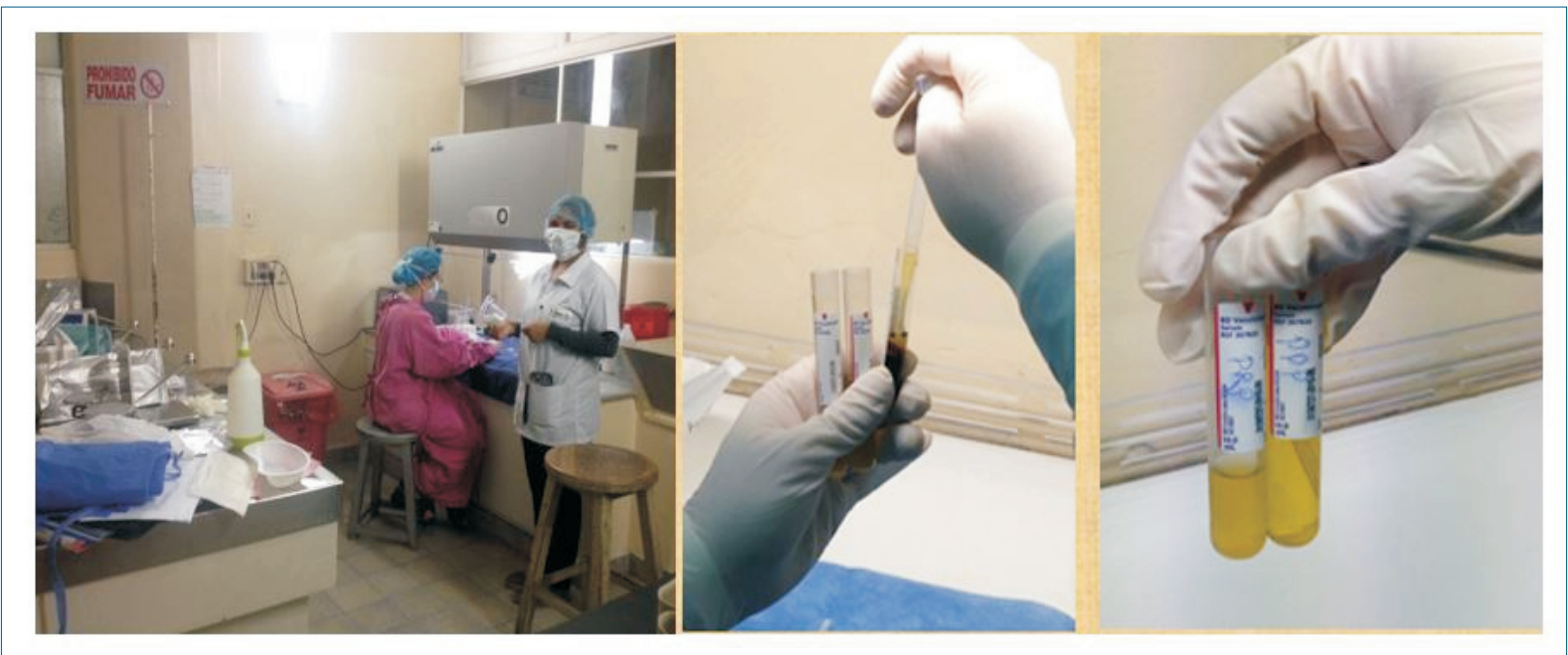

Figura 1. Obtención de PRP por método de doble centrifugación. Laboratorio de Hemoterapia del Hospital de Clínicas. Universidad de la República

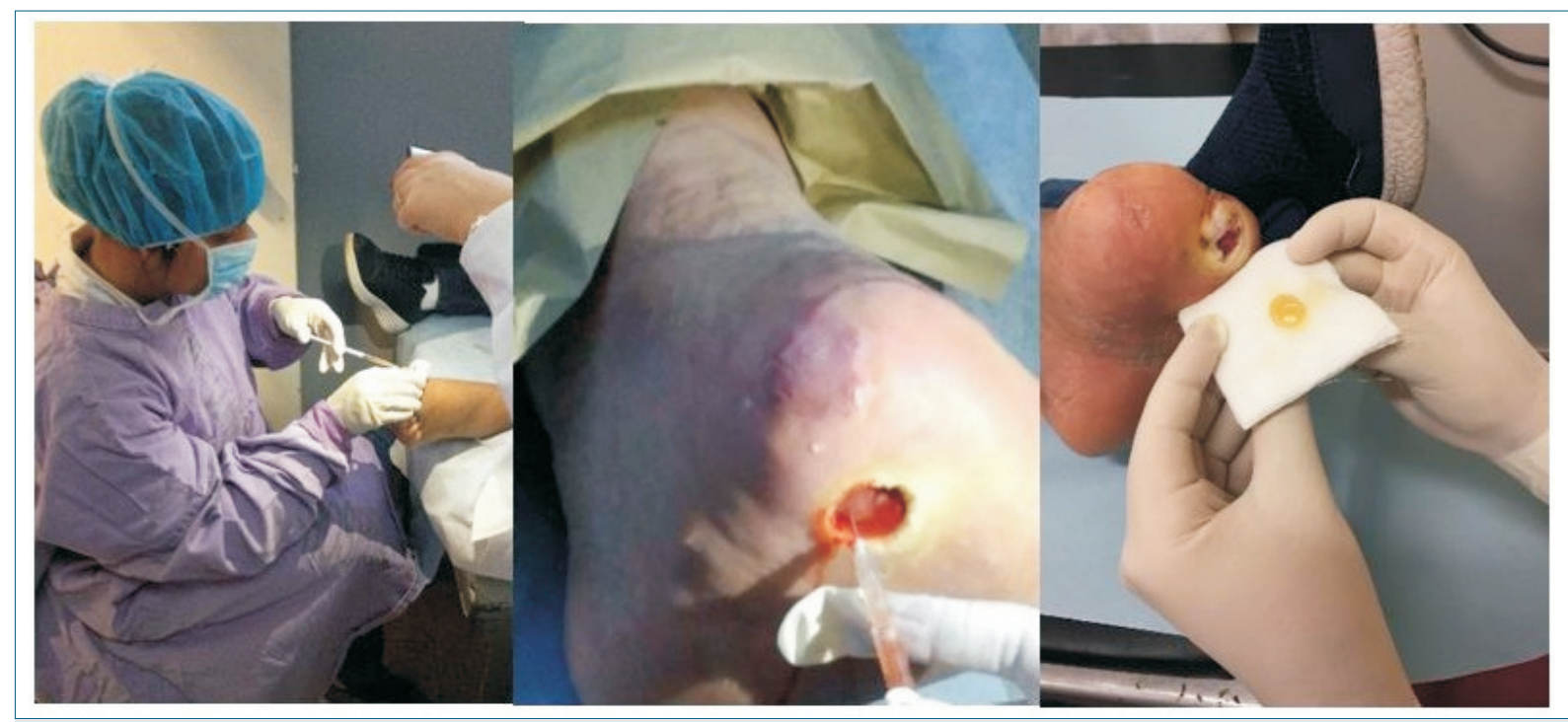

Figura 2. Aplicación del PRP. Inyección en bordes de úlcera y aplicación de gel en forma de coágulo. Pacientes con úlceras Wagner III.

vio a cada aplicación de PRP. La longitud máxima y el ancho de cada herida se determinaron usando una regla esterilizada, se realizaron dos medidas y la media se utilizó para el cálculo. Se registró el área de la úlcera en $\mathrm{cm}^{2}$ mediante Mobile Wound Analyzer (MOWA), software de análisis de imágenes de úlceras (figura 3). Se calculó el porcentaje de reducción de la úlcera utilizando la fórmula:

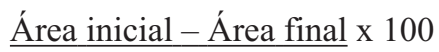
Área inicial

El protocolo de estudio se ajustó a las pautas éticas de la Declaración de Helsinki y fue aprobado por el Co- mité de Ética del Hospital Universitario. Los pacientes firmaron el consentimiento informado.

\section{Criterio de valoración y análisis estadístico}

Se consideró exitoso una reducción $\geq 70 \%$ del área de la úlcera. El crecimiento total del tejido o reepitelización sin secreción, constituyó una curación completa. La presencia de infección clínica motivó la suspensión del tratamiento. Los datos recopilados se analizaron mediante el software JMP 15.2.1 (análisis de datos) Inc. 2020, versión 15.2.1. Se calculó estadística descriptiva, medidas de resumen central y de dispersión (media, 


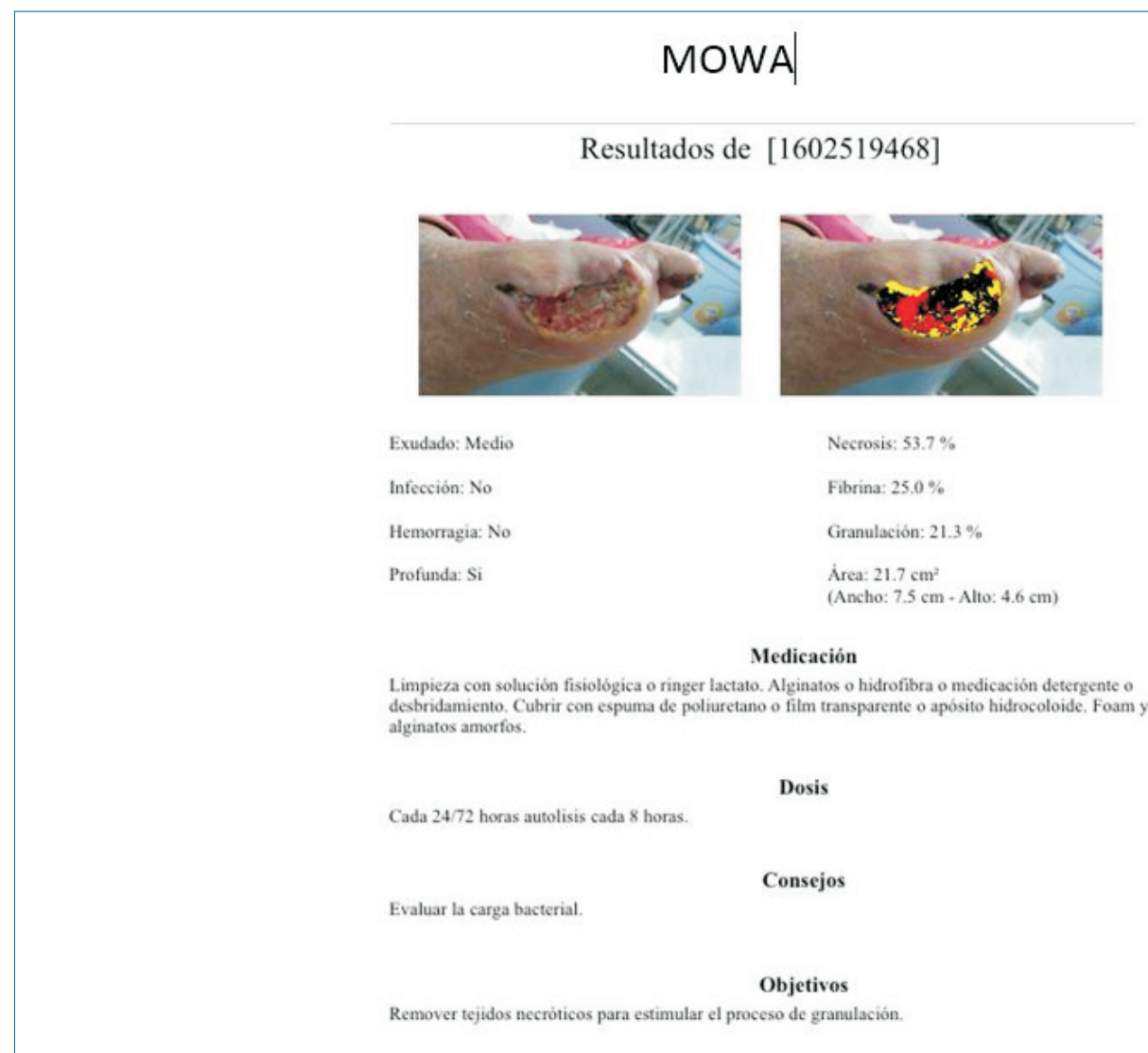

Figura 3. MOWA, software de análisis de imágenes de úlcera. Análisis planar de la imagen; evaluación cualitativa y cálculo del área.

desviación estándar e intervalos de confianza con $95 \%$ de confiabilidad) con el método binomial.

\section{Resultados}

Se realizaron 48 procedimientos en seis pacientes de forma consecutiva, el conteo plaquetario preprocedimiento de fracción F1 y del producto de PRP tuvo un enriquecimiento plaquetario dentro de lo esperado, los cultivos para aerobios y anaerobios de todos los productos realizados fueron estériles. Predominó el sexo masculino (4/6), la media de edad fue de 53 años, DE: 8,0 (42 a 63 años). Dos úlceras eran categoría II y cuatro categorías III de la clasificación de Wagner, con una media en el retraso de su cicatrización de 94 semanas (DE: 86,6; R 12-240). El control glucémico durante el seguimiento fue deficiente, con una media de HA1c: $8,33 \%$ (DE: 1,50; R 7-11). En la valoración del resultado primario a las 12 semanas, cinco pacientes tratados lograron un porcentaje de cicatrización mayor al $70 \%$ de la superficie de la úlcera con una reducción media del área de 78,83 \% (DE: 39,8) (figura 4). Antes del uso de PRP la media del área de la úlcera para todos los pacientes fue de $9,36 \mathrm{~cm}^{2}$ (DE: 6,29; R: 2,9-21,7), al final de las 12 semanas fue de $1,91 \mathrm{~cm}^{2}$ (DE: 3,52; R: 0,0-9,7) (tabla 3). Según la forma de aplicación del PRP, la respuesta obtenida fue: punción y gel redujo $75 \%, 0 \% \mathrm{y}$ $100 \%$, compactación con gel redujo $99 \%, 100 \%$ y $99 \%$ respectivamente (tabla 3). Hubo dos cicatrizaciones completas (figura 5) y dos reducciones de $99 \%$ (figura 6). Un paciente redujo el área en $75 \%$ luego del tratamiento con PRP, en una úlcera de cinco años de evolución. Otro paciente requirió una amputación por sobreinfección con Staphylococcus aureus resistente a meticilina. No observamos efectos adversos secundarios a la aplicación del PRP.

\section{Discusión}

Las UPD se caracterizan por su cronicidad y recaída ${ }^{(24)}$. La imposibilidad para curar por completo puede provocar amputaciones del pie o de la extremidad ${ }^{(25)}$. Aquellas úlceras con más de cuatro semanas de duración se consideran crónicas, siendo este un factor crítico porque la formación de biopelículas y la desecación del tejido subyacente pueden exacerbar las condiciones y di- 


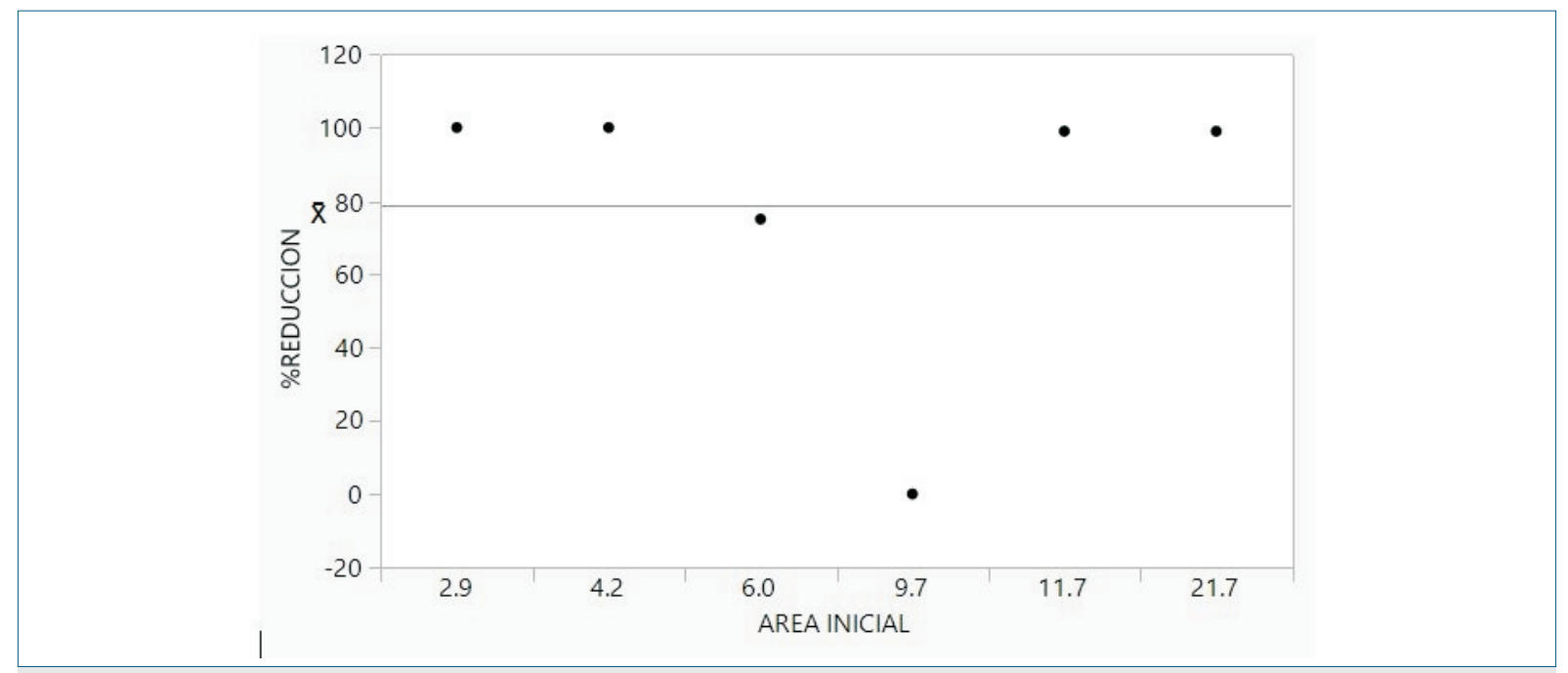

Figura 4

ficultar el proceso de epitelización ${ }^{(26)}$, derivando en una infección del pie, primera causa de amputación en UPD. El desafío es generar técnicas suplementarias para superar la insuficiencia vascular y de regeneración tisular inherentes. El uso de PRP ha mostrado una mayor cicatrización en heridas crónicas de espesor total de las extremidades inferiores ${ }^{(27-30)}$. Se han ensayado diversos métodos de centrifugación para obtener una buena concentración de PRP y sus factores de crecimiento ${ }^{(31)}$. La formulación de PRP que utilizamos logró promover la cicatrización de la úlcera tomando como punto final un período de tratamiento de 12 semanas. Nuestros pacientes no recibieron PRP en su primera visita. Para ser consistente con los resultados de nuestra Unidad de Pie, donde el $69 \%$ de los pacientes cicatrizan con una mediana de tiempo de cierre de tres meses (RIC 1-4,5) (Unidad de Pie Diabético. Hospital de Clínicas, octubre 2014 - setiembre 2016. Observación no publicada), realizamos el tratamiento convencional previo a la inclusión. Desde esta perspectiva, 31\% de nuestros pacientes se consideran refractarios al tratamiento más allá de este tiempo (10,4\% se amputan y 20,6\% continúan con una úlcera activa). En otras palabras, incluimos úlceras refractarias con un tiempo medio de retraso en la cicatrización de 94 semanas que no habían tenido beneficio con el tratamiento estándar. Sin embargo, cinco de estos pacientes con UPD Wagner II, III, reactivaron el proceso de cicatrización efectivamente en las siguientes 12 semanas, cuando agregamos el uso complementario de PRP. Esto es consistente con estudios en que se utilizó PRP para el tratamiento de úlceras refractarias $^{(32-34)}$, o que examinan la eficacia de los tratamientos utilizando el punto de corte menor a 20 semanas de terapia ${ }^{(35,36)}$. En un paciente, los fenómenos repa- rativos se encontraban ausentes o enlentecidos durante un período de cinco años y los múltiples procedimientos habían fracasado. Cuando utilizamos PRP en forma concomitante al tratamiento habitual, resultó muy favorecedor al reiniciar los procesos de reparación reduciendo el área de la úlcera en $75 \%$. En otro paciente con una amputación de dedo previa, no fue posible evitar la sobreinfección de la úlcera, requiriendo una reamputación transmetatarsiana. Se ha propuesto que PRP promueve la quimiotaxis de macrófagos y contiene una pequeña cantidad de leucocitos que sintetizan interleuquinas, previniendo infecciones ${ }^{(37)}$. Al ser un producto realizado a partir de la sangre del propio paciente, es un producto no tóxico, sin posibilidades de rechazo, alergias o contagio de enfermedades infecciosas. Una revisión que incluye 12 artículos $^{(16)}$, así como otros estudios, analizan estas complicaciones que se describen como efectos adversos de carácter leves, en general cutá$\operatorname{neos}^{(10,17,21,30)}$. No registramos eventos adversos relacionados con la aplicación de PRP en nuestros pacientes, enfatizamos las ventajas de contar con personal médico entrenado en producción de hemocomponentes bajo estándares de bioseguridad y control de calidad.

Cuando aplicamos PRP como gel, el producto semifluido mantuvo los beneficios durante el proceso de curación (figura 2). Similar a otros componentes biológicos de reparación, el gel fluye fácilmente desde una jeringa y permanece estructuralmente cohesivo. Estas características permiten una cobertura completa de las úlceras que varían en forma y profundidad, incluidas aquellas con arquitecturas geométricas o de túnel ${ }^{(38)}$, garantizando una matriz adecuada para la migración celular y el posterior contacto de célula a célula. El aumento en la concentración de los PDGF estimula significativa- 


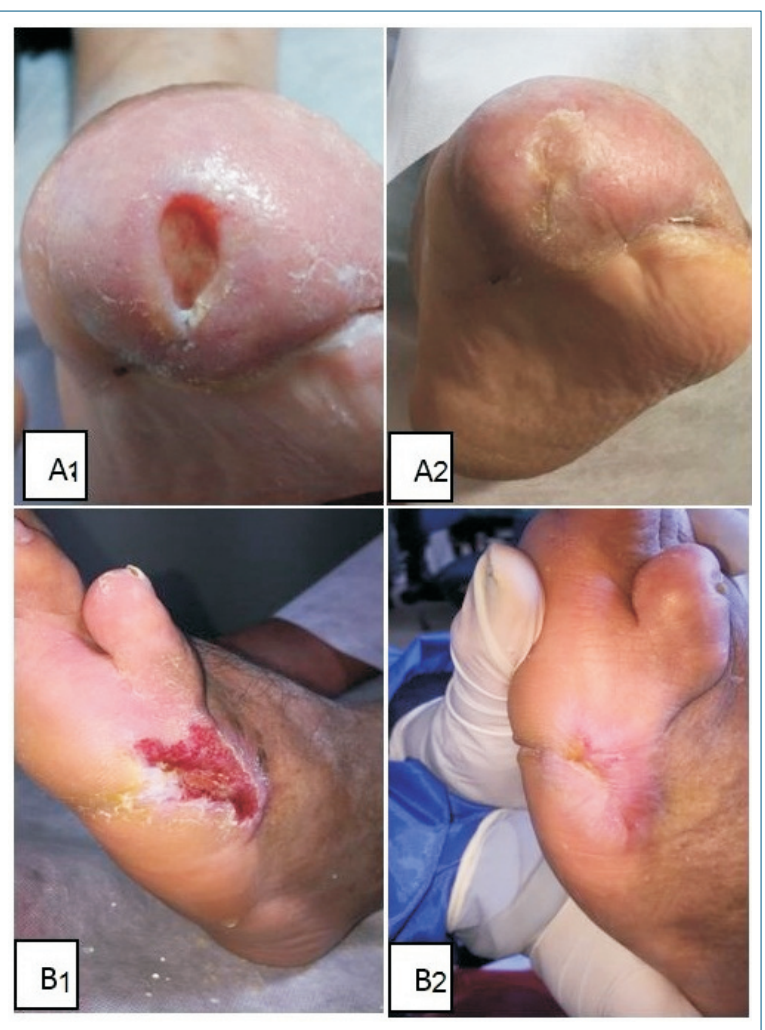

Figura 5. Pacientes representativos. (A1) Estado inicial, úlcera Wagner grado II sobre cicatriz de amputación transmetatarsiana (A2), úlcera completamente curada (día 90). (B1) Estado inicial, úlcera Wagner II del lecho de amputación en rayo del quinto dedo. (B2) Úlcera completamente curada (día 90).

mente la proliferación de fibroblastos y la expresión de colágeno tipo $\mathrm{I}^{(39)}$, estimula la granulación, la angiogénesis y la proliferación celular, promoviendo la reepitelización ${ }^{(40)}$.

Avanzar en la cicatrización de la úlcera es de gran importancia, pues los factores que cronifican la lesión, asociado a condiciones comórbidas, hace que el proceso de curación sea impredecible, aumentando la morbilidad y los costos de tratamiento.

La literatura revela escasez de estudios económicos que evalúen el tratamiento con PRP en heridas crónicas. Se ha informado que este tratamiento es coste-efectivo o incluso dominante comparado con los cuidados habituales.

$\mathrm{Su}$ eficacia determina una menor utilización de los recursos al evitar tratamientos de una úlcera activa o sus complicaciones, como ingresos hospitalarios, infecciones, amputaciones, recurrencias y segundas amputaciones ${ }^{(41,42)}$.

Un estudio que evaluó el tratamiento durante 12 semanas con PRP (aplicado dos veces por semana) demostró que es más efectivo en términos de año de vida gana-

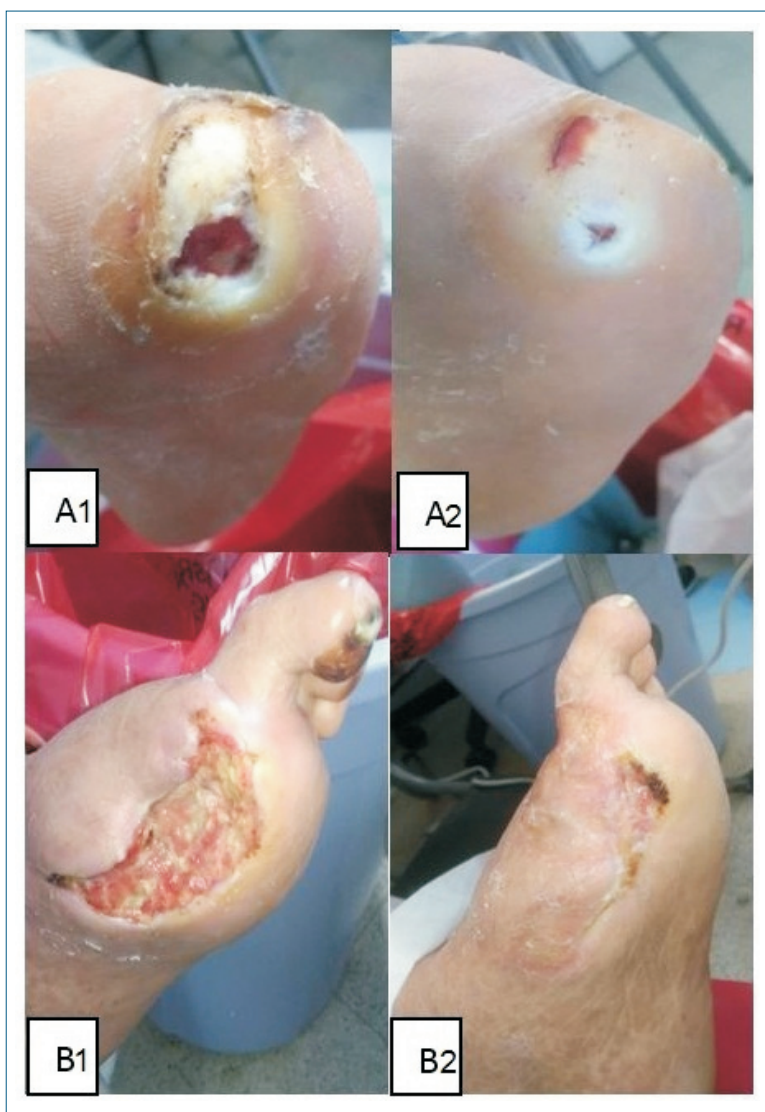

Figura 6. Pacientes representativos. (A1) Estado inicial, úlcera Wagner grado 3 sobre tercer radio del metatarsiano, en cicatriz de amputación transmetatarsiana. (A2) Úlcera cicatrizada en 99\% (día 90). (B1) Estado inicial, úlcera Wagner grado 3 del lecho de amputación en rayo del primer dedo. (B2) Se logró una reepitelización significativa en 99\% (día 90).

do ajustado por calidad (AVAC) y menos costoso (15.159 dólares) que el tratamiento estándar ${ }^{(41)}$. El costo total esperado por paciente cuya úlcera se logra epitelizar con PRP es menor que el tratamiento estándar de una úlcera refractaria $^{(12)}$.

Realizamos 48 procedimientos aplicando PRP una vez por semana durante 12 semanas, logrando epitelizar la mayoría de las úlceras que tenían una media de retraso de cicatrización de 94 semanas ( $\approx 2$ años). Un paciente debió amputarse.

En nuestra unidad el costo directo por paciente para una mediana de tiempo de epitelización de tres meses (RIC 1-4,5) asciende a 3.707 dólares. Cada procedimiento adicional en úlceras refractarias cuesta 325.910 dólares, llevando a un costo anual por úlcera que no cicatriza $\approx 15.600$ dólares/paciente. El ingreso hospitalario lo incrementa en 14.643, una amputación menor en 12.385 y una amputación mayor en 32.003 dólares. 
(Gastos en Unidad de Pie Diabético. Hospital de Clínicas, octubre 2014 - setiembre 2016. Observación no publicada).

Acortar la duración de la úlcera disminuye estos costos al evitar la necesidad de procedimientos sobre la úlcera refractaria y sus complicaciones (amputaciones). Más importante aún, disminuye la morbimortalidad, pues aquellos pacientes con UPD tienen una mortalidad a cinco años de $42 \%-48 \%{ }^{(43,44)}$.

$\mathrm{Al}$ año de seguimiento, no hubo recidiva de la lesión en cuatro pacientes y en uno de ellos persistió una úlcera de menor diámetro. Datos de un estudio que valora la recurrencia en lesiones tratadas con PRP, encontró recidivas a los 2,2 meses (rango 0,25 - 7 meses) mayor en pacientes con úlceras de evolución prolongada y descarga inadecuada del calzado poscicatrización, proponiendo que se obtendría con PRP una piel menos resistente al traumatismo en el sitio de la lesión propensa a la recidi$\mathrm{va}^{(45)}$.

Esto nos recuerda la importancia de la descarga como prevención primaria y secundaria sobre las alteraciones crónicas de la biomecánica del pie diabético que genera sitios de hiperpresión preulcerosos.

La metodología del presente trabajo (observacional, no aleatorizado) implica inevitablemente limitaciones a la hora de interpretar los datos. Sin embargo, el tratamiento fue efectivo en cinco pacientes luego de la aplicación del PRP, reduciendo el área de la úlcera en forma significativa, teniendo en cuenta que los pacientes fueron tratados previamente sin lograr avances en el proceso de cicatrización.

La efectividad se refiere a la capacidad en el mundo real de que un tratamiento proporcione un beneficio. Dado que los pacientes rara vez son sometidos a las condiciones idealizadas de los estudios controlados aleatorizados, las estimaciones de efectividad pueden ser tan útiles como las estimaciones de eficacia al momento de decidir las mejores opciones de tratamiento para los pacientes individuales ${ }^{(46)}$.

El PRP puede considerarse en úlceras con escasa o nula respuesta a los tratamientos convencionales. Presenta un costo accesible y es de fácil generación y aplicación.

Es necesario protocolizar la generación del PRP y la frecuencia de su aplicación, de esta forma podremos diseñar trabajos de investigación prospectivos que evalúen la efectividad del tratamiento asociado a PRP comparado con el tratamiento estándar. Limitaciones: necesitamos un número mayor de pacientes para poder validar el uso de PRP en el manejo de las UPD, por lo cual se siguen incluyendo pacientes. Sería interesante incluir en la investigación un soporte histológico que detalle los aspectos microscópicos de la reparación.

\section{Conclusiones}

Nuestros resultados muestran los efectos reparativos del PRP autólogo en UPD Wagner grados II y III refractarias al tratamiento estándar, mejorando las perspectivas de epitelización, sin haberse presentado efectos adversos a su uso. Se requieren más pruebas clínicas y monitoreo de seguimiento para confirmar su importancia en el manejo de las UPD difíciles de curar.

\section{Summary}

Diabetic foot ulcers imply high expenditure for both patients and the health system. Vascular impairment, growth factor inhibition and cell migration, among other factors, affect the chronicity of the condition. Platelet-derived growth factors (PDGFs) stimulate chemotaxis, neovascularization and tissue regeneration.

Objective: to evaluate effectiveness of a formulation of autologous platelet-rich plasma to complement the treatment of diabetic foot ulcer, assessing safety and adverse effects.

Material: observational study. Platelet rich plasma was applied to six patients with chronic diabetic foot ulcer Wagner II, III, with an average delayed healing of 94 weeks. PRP gel was administered by puncture technique on the lesion, once a week until the ulcer healed or for 12 weeks. Diabetic foot ulcers were analysed in regards to surface area and progression through the Mobile Wound Analyzer (MOWA) software to analyse ulcer images. The area reduction percentage was calculated comparing the initial measurement and that of week 12. The improvement criteria was defined in a reduction that is $\geq 70 \%$ of the lesion.

Results: men prevailed with an average age of 53 years \pm 7.3 . After 12 weeks of treatment an average reduction of $78 \%$ of the size of the ulcer was reported, two complete healings and two $99 \%$ healed. One patient required amputation because of uncontrolled infection.

Conclusion: the application of PRP improved tissue regeneration and shortened the duration of the ulcer, promoting healing with no adverse effects, and it likewise eliminated the need for procedures to handle chronic ulcers.

\section{Resumo}

As úlceras do pé diabético (UPD) geram alto custo para o paciente e para o sistema de saúde. A vascularização deficiente, a inibição da geração de fatores de crescimento e migração celular, entre outros fatores, influenciam sua cronicidade. Os fatores de cresci- 
mento derivados de plaquetas (PDGF) estimulam a quimiotaxia, a neovascularização e a regeneração de tecidos.

Objetivo: avaliar a eficácia de uma formulação autóloga de plasma rico em plaquetas (PRP) como modalidade complementar para o tratamento da UPD, avaliando sua segurança e efeitos adversos.

Materiais: estudo observacional. O PRP foi aplicado a 6 pacientes com UPD crônica, Wagner II, III, com um atraso médio na cicatrização de 94 semanas. Foi administrado por punção e como gel sobre a lesão uma vez por semana até o fechamento da úlcera ou por 12 semanas. As UPD foram analisadas em relação à área e sua progressão, utilizando o software de análise de imagem de úlcera Mobile Wound Analyzer (MOWA). A redução percentual da área foi calculada entre a medição inicial e a semana 12. Como critério de melhora, optou-se pela redução $\geq 70 \%$ da lesão.

Resultados: predominou o sexo masculino com média de idade de $53 \pm 7,3$ anos. Após 12 semanas de tratamento, foi relatada uma redução média no tamanho da úlcera de $78 \%$, sendo 2 com cicatrização completa e 2 em $99 \%$. Um paciente foi amputado após desenvolver infecção descontrolada.

Conclusão: a aplicação do PRP melhorou a regeneração tecidual, encurtando o tempo de duração da úlcera, promovendo sua cicatrização sem efeitos adversos, ao mesmo tempo que eliminava a necessidade de procedimentos de manejo na úlcera crônica.

\section{Bibliografía}

1. Boulton AJ. The diabetic foot: a global view. Diabetes Metab Res Rev 2000; 16(Suppl 1):S2-5. doi: 10.1002/1520-7560 (200009/10)16:1+<:aid-dmrr105>3.0.co;2-n

2. Tzeng YS, Deng SC, Wang CH, Tsai JC, Chen TM, Burnouf $T$. Treatment of nonhealing diabetic lower extremity ulcers with skin graft and autologous platelet gel: a case series. Biomed Res Int 2013; 2013:837620.

3. Guest JF, Fuller GW, Vowden P. Diabetic foot ulcer management in clinical practice in the UK: costs and outcomes. Int Wound J 2018; 15(1):43-52.

4. Lavery LA, Davis KE, Berriman SJ, Braun L, Nichols A, Kim PJ, et al. WHS guidelines update: diabetic foot ulcer treatment guidelines. Wound Repair Regen 2016; 24:112-26.

5. Wu SC, Driver VR, Wrobel JS, Armstrong DG. Foot ulcers in the diabetic patient, prevention and treatment. Vasc Health Risk Manag 2007; 3(1):65-76.

6. Raeissadat SA, Rayegani SM, Hassanabadi H, Fathi M, Ghorbani E, Babaee M, et al. Knee osteoarthritis injection choices: Platelet-rich plasma (PRP) versus hyaluronic acid (A one-year randomized clinical trial). Clin Med Insights Arthritis Musculoskeletal Disord 2015; 8:1-8. doi: 10.4137/ CMAMD.S17894.
7. Knighton DR, Doucette M, Fiegel VD, Ciresi K, Butler E, Austin L. The use of platelet derived wound healing formula in human clinical trials. Prog Clin Biol Res 1988; 266:319-29.

8. Knighton DR, Ciresi K, Fiegel VD, Schumerth S, Butler E, Cerra F. Stimulation of repair in chronic, nonhealing, cutaneous ulcers using platelet $\square$ ]derived wound healing formula. Surg Gynecol Obstet 1990; 170(1):56-60.

9. Paknejad M, Shayesteh YS, Yaghobee S, Shariat S, Dehghan M, Motahari P. Evaluation of the effect of plasma rich in growth factors (PRGF) on bone regeneration. J Dent (Tehran) 2012; 9(1):59-67.

10. Driver VR, Hanft J, Fylling CP, Beriou JM; Autologel Diabetic Foot Ulcer Study Group. A prospective, randomized, controlled trial of autologous platelet $\square$ ]rich plasma gel for the treatment of diabetic foot ulcers. Ostomy Wound Manage 2006; 52(6):68-74.

11. Anitua E, Aguirre JJ, Algorta J, Ayerdi E, Cabezas AI, Orive G, Andia I. Effectiveness of autologous preparation rich in growth factors for the treatment of chronic cutaneous ulcers. J Biomed Mater Res B Appl Biomater 2008; 84(2):415-21.

12. Li L, Wang C, Wang Y, He LP, Yang YZ, Chen LH, et al. Impact of topical application of autologous platelet $\square$ ]rich gel on medical expenditure and length of stay in hospitals in diabetic patients with refractory cutaneous ulcers. Sichuan Da Xue Xue Bao Yi Xue Ban 2012; 43(5):762-5.

13. Martinez-Zapata MJ, Martí-Carbajal AJ, Solà I, Expósito JA, Bolíbar I, Rodríguez L, et al. Autologous platelet-rich plasma for treating chronic wounds. Cochrane Database Syst Rev 2016; (5):CD006899.

14. Hu Z, Qu S, Zhang J, Cao X, Wang P, Huang S, et al. Efficacy and safety of platelet-rich plasma for patients with diabetic ulcers: a systematic review and meta-analysis. Adv Wound Care (New Rochelle) 2019; 8(7):298-308. doi:10.1089/wound.2018.0842.

15. Houdek MT, Wyles CC, Stalboerger PG, Terzic A, Behfar A, Moran SL. Collagen and fractionated platelet-rich plasma scaffold for dermal regeneration. Plast Reconstr Surg 2016; 137:1498-1506.

16. Picard F, Hersant B, Bosc R, Meningaud JP. The growing evidence for the use of platelet-rich plasma on diabetic chronic wounds: a review and a proposal for a new standard care. Wound Repair Regen 2015; 23(5):638-43. doi: 10.1111/ wrr. 12317.

17. Mohammadi MH, Molavi B, Mohammadi S, Nikbakht M, Mohammadi AM, Mostafaei S, et al. Evaluation of wound healing in diabetic foot ulcer using platelet-rich plasma gel: a single-arm clinical trial. Transfus Apher Sci 2017; 56(2):160-4. doi: 10.1016/j.transci.2016.10.020.

18. Rodriguez AE, Gisbert S, Palazón A, Alio JL. Quantification of growth factors and fibronectin in diverse preparations of platelet-rich plasma for the treatment of ocular surface disorders (E-PRP). Transl Vis Sci Technol 2020; 9(6):22. doi: 10.1167/tvst.9.6.22. 
19. Huang Y, Xie T, Cao Y, Wu M, Yu L, Lu S, et al. Comparison of two classification systems in predicting the outcome of diabetic foot ulcers: the Wagner grade and the Saint Elian Wound score systems. Wound Repair Regen 2015; 23(3):379-85. doi: 10.1111/wrr.12289.

20. Oyibo SO, Jude EB, Tarawneh I, Nguyen HC, Harkless LB, Boulton AJ. A comparison of two diabetic foot ulcer classification systems: the Wagner and the University of Texas wound classification systems. Diabetes Care 2001; 24(1):84-8. doi: 10.2337/diacare.24.1.84.

21. Kontopodis N, Tavlas E, Papadopoulos G, Pantidis D, Kafetzakis A, Chalkiadakis G, et al. Effectiveness of platelet-rich plasma to enhance healing of diabetic foot ulcers in patients with concomitant peripheral arterial disease and critical limb ischemia. Int $\mathrm{J}$ Low Extrem Wounds 2016; 15(1):45-51. doi: 10.1177/1534734615575829.

22. Furst G, Gruber R, Tangl S, Zechner W, Haas R, Mailath G, et al. Sinus grafting with autogenous platelet- rich plasma and bovine hidroxyapatite: a histomorfometric study in minipigs. Clin Oral Implants Res 2003; 14:500-8.

23. British Committee for Standards in Haematology, Blood Transfusion Task Force. Guidelines for the use of platelets transfusion. Brit J Haematol 2003; 122:10-23.

24. Kalish J, Hamdan A. Management of diabetic foot problems. J Vasc Surg 2010; 51(2):476-86.

25. Veves A, Falanga V, Armstrong DG, Sabolinski ML; Apligraf Diabetic Foot Ulcer Study. Graftskin, a human skin equivalent, is effective in the management of noninfected neuropathic diabetic foot ulcers: a prospective randomized multicenter clinical trial. Diabetes Care 2001; 24(2): 290-5.

26. Reyzelman A, Crews RT, Moore JC, Moore L, Mukker JS, Offutt S, et al. Clinical effectiveness of an acellular dermal regenerative tissue matrix compared to standard wound management in healing diabetic foot ulcers: a prospective, randomised, multicentre study. Int Wound J 2009; 6(3):196208.

27. Montón Echeverría J, Pérez Redondo S, Gómez Bajo GJ. Experiencia clínica en el empleo de factores de crecimiento autólogos obtenidos de plasma rico en plaquetas. Cir Plást Iberolatinoam 2007; 33(3):155-62. Disponible en: http://scielo.isciii.es/scielo.php?script=sci_arttext\&pid=S0376-78922007000300002\&lng=es.[Consulta: 14 junio 2019].

28. Scimeca CL, Bharara M, Fisher T, Kimbriel H, Armstrong D. Novel use of platelet-rich plasma to augment curative diabetic foot surgery. J Diabetes Sci Technol 2010; 4(5):1121-6.

29. Tzeng YS, Deng SC, Wang CH, Tsai JC, Chen TM, Burnouf $\mathrm{T}$. Treatment of nonhealing diabetic lower extremity ulcers with skin graft and autologous platelet gel: a case series. Biomed Res Int 2013; 2013:837620.

30. Li L, Chen DW, Wang C, Yuan NB, Wang Y, He LP, et al. Autologous platelet-rich gel for treatment of diabetic chronic refractory cutaneous ulcers: A prospective, randomized clinical trial. Wound Repair Regen 2015; 23(4):495-505.

31. Yuan N, Wang C, Wang Y, Yu T, Long Y, Zhang X, Ran X. Preparation of autologous platelet-rich gel for diabetic refractory dermal ulcer and growth factors analysis from it. Zhongguo Xiu Fu Chong Jian Wai Ke Za Zhi 2008; 22(4):468-71.

32. Frykberg RG, Driver VR, Carman D, Lucero B, BorrisHale C, Carelyn P. Chronic wounds treated with a physiologically relevant concentration of plateletrich plasma gel: a prospective case series. Ostomy Wound Manage 2010; 56(6):36-44.

33. Cervelli V, De Angelis B, Lucarini L, Spallone D, Balzani A, Palla $\mathbf{L}$, et al. Tissue regeneration in loss of substance on the lower limbs through use of platelet-rich plasma, stem cells from adipose tissue, and hyaluronic acid. Adv Skin Wound Care 2010; 23(6):262-72.

34. Sakata J, Sasaki S, Handa K, Uchino T, Sasaki T, Higashita $\mathbf{R}$, et al. A retrospective, longitudinal study to evaluate healing lower extremity wounds in patients with diabetes mellitus and ischemia using standard protocols of care and platelet-rich plasma gel in a Japanese wound care program. Ostomy Wound Manage 2012; 58(4):36-49.

35. Wieman TJ, Smiell JM, Su Y. Efficacy and safety of a topical gel formulation of recombinant human platelet-derived growth factor-BB (becaplermin) in patients with chronic neuropathic diabetic ulcers: a phase III randomized placebo-controlled double-blind study. Diabetes Care 1998; 21:822-7.

36. Gentzkow GD, Iwasaki SD, Hershon KS, Mengel M, Prendergast JJ, Ricotta JJ, Steed DP, Lipkin S. Use of dermagraft, a cultured human dermis, to treat diabetic foot ulcers. Diabetes Care 1996; 19:350-4.

37. Chen L, Wang C, Liu H, Liu G, Ran X. Antibacterial effect of autologous platelet-rich gel derived from subjects with diabetic dermal ulcers in vitro. J Diabetes Res 2013; 2013:269527.

38. Brigido SA, Schwartz E, McCarroll R, Hardin-Young J. Use of an acellular flowable dermal replacement scaffold on lower extremity sinus tract wounds: a retrospective series. Foot Ankle Spec 2009; 2(2):67-72.

39. Cho JW, Kim SA, Lee KS. Platelet-rich plasma induces increased expression of G1 cell cycle regulators, type I collagen, and matrix metalloproteinase- 1 in human skin fibroblasts. Int J Mol Med 2012; 29:32-6.

40. Pietramaggiori G, Scherer SS, Mathews JC, Gennaoui T, Lancerotto L, Ragno G, et al. Quiescent platelets stimulate angiogenesis anddiabetic wound repair. J Surg Res 2010; 160:169-77.

41. Dougherty EJ. An evidence-based model comparing the costeffectiveness of platelet-rich plasma gel to alternative therapies for patients with nonhealing diabetic foot ulcers. Adv Skin Wound Care 2008; 21(12):568-75. 
42. Kantor J, Margolis DJ. Treatment options for diabetic neuropathic foot ulcers: a cost-effectiveness analysis. Dermatologic Surg 2001; 27(4):347-51.

43. International Diabetes Federation; Ibrahim A, Jude E, Langton K, Martinez-De Jesus FR, Harkless LB, Gawish H, et al. IDF Clinical Practice Recommendation on the Diabetic Foot - 2017: a guide for health care professionals. Brussels: IDF, 2017. Disponible en: https://www. idf.org/e-library/guidelines/119-idf-clinical-practice-recommendations-on-diabetic-foot-2017.html. [Consulta: 14 junio 2019].

44. Martins-Mendes D, Monteiro-Soares M, Boyko EJ, Ribeiro M, Barata $\mathbf{P}$, Lima $\mathbf{J}$, et al. The independent contri- bution of diabetic foot ulcer on lower extremity amputation and mortality risk. J Diabetes Complicat 2014; 28(5): 632-8.

45. Steed DL, Edington HD, Webster MW. Recurrence rate of diabetic neurotrophic foot ulcers healed using topical application of growth factors released from platelets. Wound Repair Regen 1996; 4(2):230-3. doi: 10.1046/j.1524-475X. 1996.40210.x

46. Margolis DJ, Kantor J, Santanna J, Strom BL, Berlin JA. Effectiveness of platelet releasate for the treatment of diabetic neuropathic foot ulcers. Diabetes Care 2001; 24(3):483-8. doi: $10.2337 /$ diacare.24.3.483.

\section{Contribución de autores}

Pablo Orellano, 0000-0002-5080-5961. Concepción; diseño, ejecución, análisis de datos, interpretación de los resultados, redacción. Matias Torres Negreira, 000000031098 3680. Concepción, diseño, ejecución, revisión crítica.

Agustín Colombo, 0000-0002-3812-9286. Ejecución, análisis de resultados, revisión crítica.

Natalia Lamela, 0000-0002-2582-7325. Concepción, diseño, ejecución, recolección de datos.

Daniela Saliwonczyk, 000000026219 9698. Ejecución, recolección de datos.

Maximiliano Berro, 0000-0002-7513-5466. Revisión, análisis crítico.

Gabriela Sierra, 0000-0002-2104-2395. Ejecución, recolección de datos.

Ismael Rodríguez, 0000-0002-3361-7117. Concepción revisión, análisis crítico.

Lilian Vucovich, 0000-0002-0801-8649. Ejecución, revisión crítica. 